\title{
Coronary artery disease is a significant cause of morbidity and premature death in patients with systemic lupus erythematosus
}

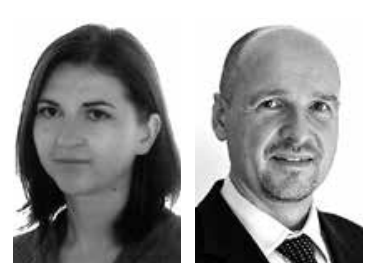

\author{
Anna Karpińska, Michał Ciurzyński \\ Department of Internal Medicine and Cardiology, Medical University of Warsaw, Poland
}

Systemic lupus erythematosus (SLE) is a systemic chronic disease in which impaired function of immunocompetent cells leads to inflammation and internal organ failure. In the course of SLE most patients develop cardiological complications, which are among the most dangerous complications of SLE. Cardiovascular manifestations of SLE include accelerated coronary heart disease (CHD), pericarditis, myocarditis, valvular disease, Libman-Sacks nonbacterial endocarditis (the most characteristic cardiac manifestation of SLE), conduction abnormalities, pulmonary hypertension, thromboembolic events, hypertension and heart failure. In recent years, it has been widely appreciated that premature atherosclerosis is a major cause of death in patients with SLE, especially in young or middle-aged women, for whom the background rate of CHD outcomes is very low.

Despite improvements in overall lupus mortality, due to better possibilities of diagnosis and treatment, the risk of mortality seems to be 3 to 5 times greater in patients with SLE compared with the general population [1]. Furthermore, a large international cohort study suggested that although standardized all-cause mortality rates (SMR) for SLE decreased from 4.9 (in 1970-1979) to 2.0 (in 1990-2001), the SMR for cardiovascular disease (CVD) in SLE did not decrease over the same period [2].

An increased risk of cardiovascular events among patients with SLE has been confirmed in multiple studies. Bernatsky et al. compared the mortality in an SLE cohort with general population mortality rates. In 23 centers, 9547 patients (90\% of the patients were female) were observed for an average of 8.1 years. During the observation 1,255 deaths occurred and the most common types of deaths (313 patients) not directly attributed to SLE were deaths due to circulatory disease (including all types of heart disease, arterial disease and cerebrovascular events - strokes). The SMR for death due to circulatory disease was 1.7 (95\% Cl 1.5-1.9) [2]. In studies of young necropsy patients with SLE substantial atherosclerosis was present in up to half $[3,4]$. Manzi et al. [5] found that women with SLE in the 35 to 44year age group were over 50 times more likely to have a myocardial infarction (MI) compared with their healthy counterparts in the Framingham Offspring Study. The dramatic increase in CHD in young patients with SLE has been widely recognized and is an important concern of current clinical research, but the pathogenesis is still unknown. Indeed, traditional risk factors such as hypertension, hypercholesterolemia, diabetes mellitus, tobacco use, obesity, family history of CHD and sedentary lifestyle are common among patients with SLE. Not without significance is the treatment used in SLE, such as corticosteroids, which contribute to the damage of the cardiovascular system. What is of importance is that in SLE patients traditional risk factors are not believed to fully account for the increased atherosclerosis. Rahman et al. [6] found that SLE patients with a cardiac event have fewer traditional risk factors than non-SLE patients with premature CHD. In women with SLE the mean number of CHD risk factors per cardiac event was $2.0 \pm 0.77$ vs. 2.90 \pm 1.19 for the comparison group ( $p=0.0008)$. Similar findings were reported in men with SLE. The mean number of CHD risk factors was $1.87 \pm 0.83$ vs. $2.73 \pm 0.99$ in the comparison group $(p=0.016)$ [6]. This phenomenon can be attributed to coexisting traditional risk factors for atherosclerosis, and the use of corticosteroids, but also might be the result of other autoimmune and inflammatory mechanisms that are aggravated by SLE.

Many components of the immune system are involved in the pathologic processes underlying the development of atherosclerosis: macrophages, $T$ cells, 
autoantibodies (anti-nuclear antibodies, anti-cardiolipin antibodies, anti-C-reactive protein antibodies, anti-endothelial cell antibodies), autoantigens and cytokines that are secreted by cells within atherosclerotic plaques, including interleukin (IL)-1, IL-2, IL-6, IL-8, IL-12, IL-10, tumor necrosis factor, interferon- $\gamma$ and platelet-derived growth factor.

Circulating immune complexes accumulate in the vessel wall. The vascular endothelium undergoes a series of inflammatory changes that result in endothelial dysfunction, which is an early stage in the process of atherosclerotic plaque formation. Myocardial infarction occurs when one of these plaques ruptures or when platelets aggregate, leading to narrowing of the arterial lumen. However, MI in patients with SLE may rarely be due to thrombosis in the coronary artery without significant atherosclerosis or result from coronary vasculitis or arterial emboli [7]. Multiple potential biomarkers have been associated either with cardiovascular events or with prospective longitudinal measures of subclinical atherosclerosis. Most of them (antiphospholipid antibodies, CRP/hs-CRP, erythrocyte NO production, paraoxonase 1, adiponectin, annexin A5, ADMA, C3 and C5a, E-selectin, FAB4, ICAM, leptin, OxLDL and Ox-PAPC, autoantibodies to OxLDL, antioxidized phosphatidylserine, anti-PC antibodies, piHDL, TNF-a, sTWEAK, VCAM, VWF, whole blood viscosity) are still in the preliminary phases of investigation [8]. More trials need to be done for identification of SLE-specific biomarkers that could easily recognize patients at future risk for atherosclerosis. Studies using noninvasive screening tests - intima-media thickness (IMT), ankle-brachial index (ABI), assessment of vascular elasticity by measurement of pulse wave velocity (PWV), assessment of endothelial dysfunction by measurement of brachial artery reactivity in response to changes in blood flow caused by passive congestion: flow-mediated vasodilatation (FMD) or EndoPAT - support an increased prevalence of asymptomatic atherosclerosis in patients with SLE. One of several similar studies confirms the presence of both endothelial dysfunction and subclinical atherosclerosis in SLE patients. Mean IMT in all SLE patients (0.83-0.11 mm) was significantly higher when compared to controls (0.66 $\pm 0.04 \mathrm{~mm}, p<0.05)$. Mean FMD was significantly smaller in SLE patients $(2.98 \pm 1.58 \%)$ when compared to controls $(10.83 \pm 2.02 \%, p<0.05)$ [9]. However, it is not recommended to use these tests to screen asymptomatic patients with SLE for CHD in the absence of other traditional risk factors for CHD. In patients with SLE and nonspecific complaints (such as chest discomfort and/or dyspnea), with long durations of active disease or other traditional risk factors, screening for CHD with exercise or pharmacologic stress testing is advis- able. Patients with SLE and symptomatic CHD should be treated aggressively, as in patients without SLE who have diabetes or chronic kidney disease. Primary and secondary prevention strategies directed at hypertension, hypercholesterolemia and obesity as well as other known CHD risk factors should be routinely employed in the management of patients with SLE. Statins (with their pleiotropic effects including antiinflammatory, antithrombotic, and plaque-stabilizing activities) should be used when hypercholesterolemia is inadequately controlled by lifestyle and dietary modification. Because glucocorticosteroids contribute to CHD risk factors by worsening hypertension, hyperlipidemia, and diabetes, they should be used only when properly indicated and in the lowest dosage when no other alternative treatment is available. The impact of modulators of lupus disease activity (such as antimalarial therapy, azathioprine, and mycophenolate mofetil) is still not well known, and larger prospective studies will be needed to examine the effect of new medications on CHD. Beneficial interventions also include not smoking, exercising regularly and achieving goal blood pressure in those with hypertension (angiotensin-converting enzyme inhibitors are generally the drugs of choice in patients with renal disease; calcium channel blockers may be useful in patients with coexisting Raynaud phenomenon or pulmonary hypertension).

In summary, the prevalence of atherosclerosis is higher in patients with SLE and occurs at an earlier age. In recent years, it has been confirmed in multiple studies that CHD is a major cause of death in patients with SLE. Clinicians who treat patients with SLE should be aware of the importance of risk factor reduction in the prevention and treatment of CHD. Therefore, early identification and treatment of lupus patients with CHD is of clinical importance to reduce morbidity and mortality.

The authors declare no conflict of interest.

\section{References}

1. Olesińska M. Toczeń rumieniowaty układowy. In: Antczak A, Myśliwiec M, Pruszczyk P, et al. (ed.) Wielka interna. Reumatologia. Medical Tribune, Warszawa 2011; 97-113.

2. Bernatsky S, Boivin JF, Joseph L, et al. Mortality in systemic lupus erythematosus. Arthritis Rheum 2006; 54: 2550-2557.

3. Bulkley $\mathrm{BH}$, Roberts WC. The heart in systemic lupus erythematosus and the changes induced in it by corticosteroid therapy. A study of 36 necropsy patients. Am J Med 1975; 58 : 243-264.

4. Haider YS, Roberts WC. Coronary arterial disease in systemic lupus erythematosus; quantification of degrees of narrowing in 22 necropsy patients ( 21 women) aged 16 to 37 years. Am J Med 1981; 70: 775-781. 
5. Manzi S, Meilahn EN, Rairie JE, et al. Age-specific incidence rates of myocardial infarction and angina in women with systemic lupus erythematosus: comparison with the Framingham Study. Am J Epidemiol 1997; 145: 408-415.

6. Rahman P, Urowitz MB, Gladman DD, et al. Contribution of traditional risk factors to coronary artery disease in patients with systemic lupus erythematosus. J Rheumatol 1999; 26: 2363-2368.

7. Kutom AH, Gibbs HR. Myocardial infarction due to intracoronary thrombi without significant coronary artery disease in systemic lupus erythematosus. Chest 1991; 100: 571-572.

8. McMahon M, Skaggs B. Pathogenesis and Treatment of Atherosclerosis in Lupus. Rheum Dis Clin N Am 2014; 40: 475495.

9. Pompilian V, Bălănescu PC, Bălănescu E, Badea C. Annexin A5 as independent predictive biomarker for subclinical atherosclerosis and endothelial dysfunction in systemic lupus erythematosus patients. Clin Lab 2013; 59: 359-367. 